\title{
Nonlinear optical properties of stilbene and azobenzene derivatives containing azaphosphane groups
}

\author{
PRAKASH CHANDRA JHA, Y. ANUSOOYA PATI and S. RAMASESHA* \\ Solid State and Structural Chemistry Unit, Indian Institute of Science, Bangalore 560 012, India
}

\begin{abstract}
We have studied the nonlinear optical (NLO) properties of some donor-acceptor molecules with stilbene and azobenzene molecules as backbone. We have used the nitro group as the acceptor and azaphosphane $\left(\mathrm{R}_{3} \mathrm{P}=\mathrm{N}-\right)$ as the donor group. To study the effect of variation of NLO properties, we have replaced the substituents (Rs) connected to the phosphorus atom by methyl, amine and phenyl groups. We find that both first-order polarizability and hyperpolarizabilities are larger for stilbene derivatives and is maximum for the phenyl substitution. Second-order polarizability is higher for methyl substitution. We have also obtained the two-photon absorption cross-section for these molecules. We find that both one-photon and two-photon absorption cross-sections are maximum for the same excited state (first excited state in the case of stilbene and second excited state in the case of azobenzene derivatives).
\end{abstract}

\section{Introduction}

Conjugated organic materials containing electron donors (D) and electron acceptors (A) are known to exhibit very large nonlinear optical responses particularly with respect to second harmonic generation (SHG) and electro-optical effects [1-7]. Organic and organicinorganic hybrid push-pull materials displaying large optical nonlinearities have found a wide range of applications in devices for optical data storage, optical information processing and electro-optic or all optic switches [1-7]. The focus of the research in this area is in finding materials with excellent solubility, adequate thermal stability and desired optical properties, such as large nonlinearity, fast response time and low transmission loss [1]. In this context several different classes of electron donor-acceptor groups have been attached to the backbone of conjugated molecules, like heterocycles, unsaturated aliphatic hydrocarbon linkages, such as vinyl and ethyl, and aromatic hydrocarbons, such as benzene and stilbene. It is well known in the literature that the donor and acceptor substituents provide the requisite ground-state charge asymmetry, while the $\pi$-conjugation provides a pathway for the transfer and redistribution of electric charges under the influence of electric fields [1-15]. The theoretical as well as experimental investigations of the structure-property relationship provide pertinent information for the

\footnotetext{
*Corresponding author. Email: ramasesh@sscu.iisc.ernet.in
}

engineering of novel molecules. Such investigations help in finding the relation between the nonlinear optical response function such as hyperpolarizability, two-photon absorption cross-section and molecular features, such as the strengths of donor and acceptor groups, and nature, as well as the extent of the $\pi$-conjugation. For example, it has been shown that the first-order hyperpolarizability $\beta$ can be increased by increasing the strength of the donor-acceptor groups as well as length of the conjugation. However, it has also been shown from a two-level model that there is an optimal combination of the donor-acceptor groups that will result in a maximum $\beta[16,17]$. Additionally for a given pair of donor-acceptor groups there is an optimal length of conjugation for which $\beta$ is maximum $[17,18]$. Davydov et al. have proposed that electron donor and electron acceptor groups attached to benzene and stilbene should result in molecules with large $\beta$ [19]. The effect of push-pull groups is to produce a large difference in the dipole moment between the ground and excited states of the molecules, which in turn will result in a large $\beta$.

According to the two state model of Oudar and Chemla, to optimize the nonlinear optical response and its relationship between the nature of donor-acceptor groups and the structure of the $\pi$-conjugating spacers, a large number of new and novel combinations of the electron donor and acceptor groups have been investigated [20]. Generally, the active materials used for such purpose involve a host polymeric backbone, functionalized with nonlinear chromophores either as a guest 
molecule or via covalent bonding to the polymer chain. In both cases, the realization of a non-centrosymmetric active medium requires appropriate alignment of the dipole moments of the push-pull chromophores. The process of dipole orientation is generally achieved by heating a polymer matrix containing the chromophore around the glass transition temperature $\left(T_{\mathrm{g}}\right)$ while maintaining an applied electric field during the cooling process. But the major problem associated with such materials is the process of structural relaxation of the chromophores, which, in turn, leads to head-to-tail orientation of the dipoles resulting in a loss of the desired nonlinear optic (NLO) activity. Hence, we need to look for materials with high $T_{\mathrm{g}}$, which, in turn, render high chemical and thermal stability to the backbone with incorporated NLO chromophores [21]. As the chemical and thermal stability of the incorporated NLO chromophores play a significant role in the overall design of NLO devices, development of thermally robust new chromophores is of paramount importance. In this context, the use of azaphosphanes as a new class of electron donors in the design and development of NLO optical materials has been quite promising [22] Azaphosphanes are supposed to have the highest thermal stability (stable up to $335^{\circ} \mathrm{C}$ ) among all the chromophores reported for organic nitro acceptor NLO compounds [22]. Besides, this particular electron donating group has a number of useful features for practical device purposes. The azaphosphane group can be covalently linked to conjugated backbones over a broad range of experimental conditions (such as concentration range) in a controlled manner. This in turn implies that most of the physical properties, such as mechanical, optical and electrical, can be tailored by changing the nature and concentration of the side groups attached to the polymer chain by suitable molecular engineering. Furthermore, even by using polar side groups significant second-order nonlinear optical responses have been achieved thereby suggesting that azaphosphanes are promising candidates for the design of molecules that can be employed in device fabrication [23]. Although, this electron donating group belongs to a new class of electron donor, which can donate up to three electrons to electron acceptors, the powerful electron donating ability of azaphosphanes has already been used to stabilize transition metals in their high oxidation states [24]. In addition to the electron donating ability, there is a possibility of using a wide combination of organic functionalities that can be attached to the phosphorus centre, which in turn will provide opportunities for fine-tuning solubility, thermal and optical properties of this new generation of chromophores.

In the present study, the use of azaphosphanes as electron donors in the chemical design of a new generation of dipolar push-pull (donor-acceptor) compounds have been theoretically evaluated for their NLO response. The chemical architecture used in this study include stilbene, as well as azobenzene, which can be considered as a pseudo-stilbene molecule as they contain an azo-linkage $(\mathrm{N}=\mathrm{N})$ between two phenyl groups. An enormous number of experimental and theoretical studies have focused on the nonlinear optical properties of such types of conjugated molecules. We also present results for the combined effect of changing the electron donating groups attached to phosphorous, involved in the $\mathrm{P}=\mathrm{N}$ bond, with the nitro group as the acceptor. Besides, we have studied the effect of extended conjugation by introducing phenyl groups as substituents on the phosphorous atom. The substituents we have considered are mono, di and triphenyl substitution, trimethyl and triamino substitutions, besides, of course the unsubstituted system. The molecular structures are shown in figure 1 . This type of efficient donor-acceptor groups and extended conjugation are expected to enhance electronic interaction and could generate highly polarizable charge transfer, with asymmetric electron distribution.

\section{Methodology}

In the simplest models, the frequency dependent linear and nonlinear optical coefficient calculations rely on the knowledge of transition dipole moments between the ground state and the key charge transfer (CT) excited states, the energy gap to the CT state, the dipole moments for the ground state and the CT state. The sum over states (SOS) method which is the most widely used method incorporates a larger number of excited states compared to the simplest models. However, the SOS method involves the uncontrolled approximations of truncating the excitation spectrum of the molecule which leads to convergence problems especially in the computation of higher order NLO coefficients. Within the SOS formalism, it is usually computationally prohibitive to include all the excited states within the chosen Hilbert space.

However, it is possible to incorporate all the excited states by the correction vector method implemented in the ZINDO package [25] of Ramasesha et al. [26], without explicitly computing all the eigenvalues and eigenfunctions of the configuration interaction (CI) Hamiltonian. It is generally believed that in CI implementation of ZINDO, it is enough to include only singly excited configurations (SCI), if we are interested just in the polarizability $\alpha$ and the first-order hyperpolarizability $\beta$. The reason put forth to explain 

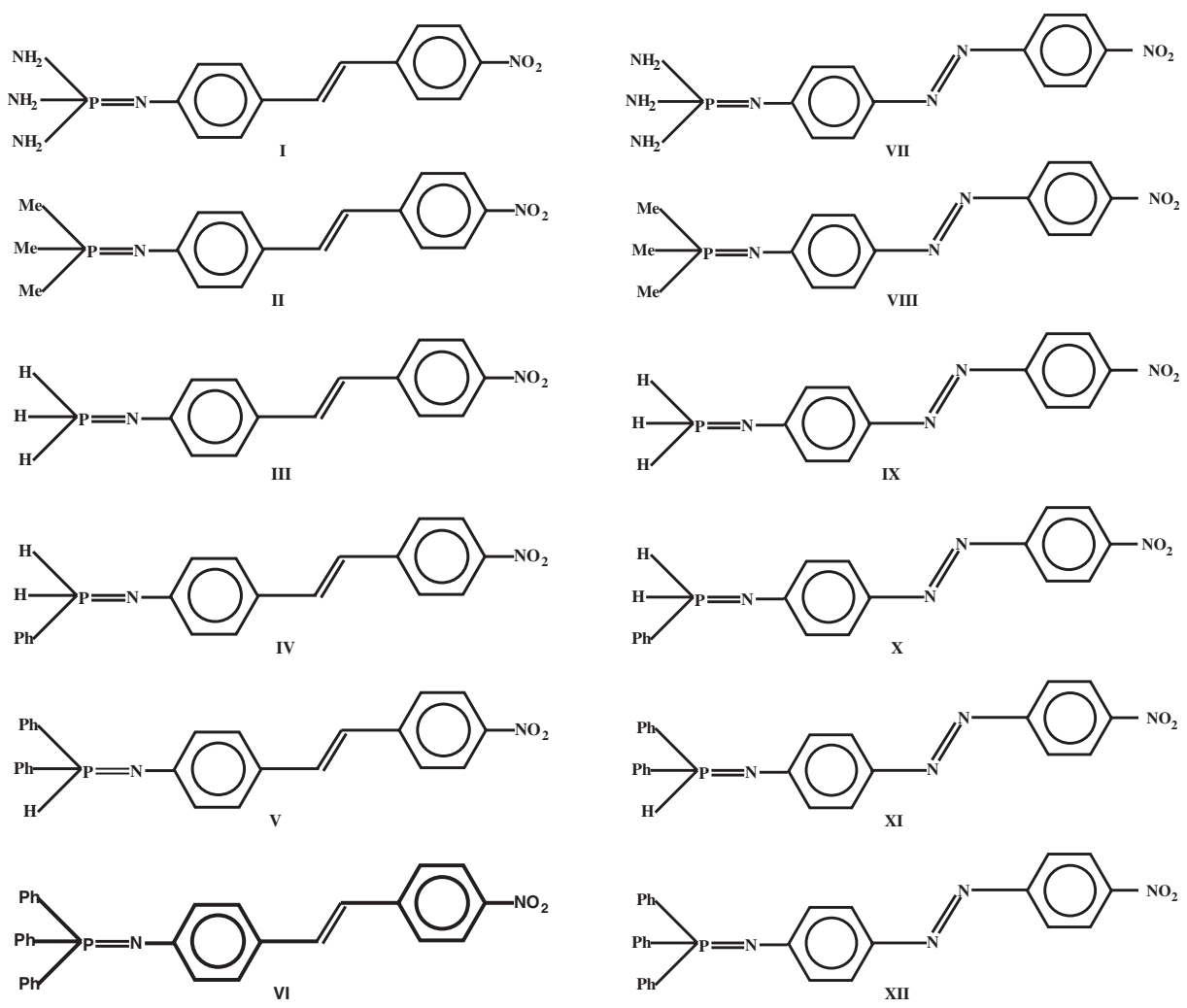

Figure 1. Structures of azaphosphane derivatives of stilbene (I to VI) and corresponding derivatives of azobenzene (VII to XII) studied.

the use of SCI is that the first nonlinear optical coefficients are derived from second-order perturbation theory involving one electron excitations. In fact, this approach has been used quite extensively in several of the earlier studies by different groups and was found to provide excitation energies and dipole matrix elements in good agreement with experiment. The observation that the first-order hyperpolarizability is well reproduced by SCI is purely empirical. In simple conjugated hydrocarbons such as polyacetylene and similar polyene, SCI calculations have been relied upon to compute $\alpha$ and $\beta$. To compute accurately the second-order hyperpolarizability $(\gamma)$, there is enough evidence to show that SCI is not enough. In our calculation we have used a single and double excitation method (SDCI). A SDCI method is more sophisticated compared to the SCI because it treats the repulsive interaction between a given pair of electrons. In fact, it is well known in the literature that, in the case of $\gamma$, to obtain the correct sign we should include at least the double excitation in addition to the single excitation.

The calculations of NLO properties follow the correction vector (CV) approach of Soos and Ramasesha [27], incorporated into the ZINDO package by Ramasesha et al. [26]. It involves solving for the vectors $\phi_{i}^{(1)}\left(\omega_{1}\right)$ and $\phi_{i j}^{(2)}\left(\omega_{1}, \omega_{2}\right)$, which are defined as

$$
\begin{gathered}
\left(\mathbf{H}-E_{\mathrm{G}}+\hbar \omega_{1}+\mathrm{i} \Gamma\right)\left|\phi_{\mathrm{i}}^{(1)}\left(\omega_{1}\right)\right\rangle=\tilde{\mu}_{i}|G\rangle, \\
\left(\mathbf{H}-E_{\mathrm{G}}+\hbar \omega_{2}+\mathrm{i} \Gamma\right)\left|\phi_{\mathrm{ij}}^{(2)}\left(\omega_{1}, \omega_{2}\right)\right\rangle=\tilde{\mu}_{j}\left|\phi_{i}^{(1)}\left(\omega_{1}\right)\right\rangle,
\end{gathered}
$$

where $\mathbf{H}$ is the Hamiltonian matrix in the chosen manybody basis, $E_{\mathrm{G}}$ is the ground state energy, $\omega_{1}, \omega_{2}$ are the frequencies and $\tilde{\mu}_{i}$ is the $i$ th component of the dipole displacement operator, $\tilde{\mu}_{i}=\hat{\mu}_{i}-\left\langle G\left|\hat{\mu}_{i}\right| G\right\rangle$ and $\Gamma$ is the average lifetime of the excited states. It can be shown that $\phi_{i}^{(1)}\left(\omega_{1}\right)$ and $\phi_{i j}^{(2)}\left(\omega_{1}, \omega_{2}\right)$ expressed in the basis of the eigenstates of the Hamiltonian $|R\rangle$ are given by

$$
\begin{aligned}
& \left|\phi_{i}^{(1)}\left(\omega_{1}\right)\right\rangle=\sum_{R} \frac{\left\langle R\left|\tilde{\mu}_{i}\right| G\right\rangle}{E_{R}-E_{\mathrm{G}}+\hbar \omega_{1}+\mathrm{i} \Gamma}|R\rangle, \\
& \left|\phi_{i j}^{(2)}\left(\omega_{1}, \omega_{2}\right)\right\rangle \\
& =\sum_{S} \sum_{R} \frac{\left\langle S\left|\tilde{\mu}_{j}\right| R\right\rangle\left\langle R\left|\tilde{\mu}_{i}\right| G\right\rangle}{\left(E_{R}-E_{\mathrm{G}}+\hbar \omega_{1}+\mathrm{i} \Gamma\right)\left(E_{S}-E_{\mathrm{G}}+\hbar \omega_{2}+\mathrm{i} \Gamma\right)}|S\rangle .
\end{aligned}
$$

Therefore $\phi_{i}^{(1)}\left(\omega_{1}\right)$ and $\phi_{i j}^{(2)}\left(\omega_{1}, \omega_{2}\right)$ can be readily used to compute linear and nonlinear frequency dependent 
polarizabilities within the Pariser-Parr-Pople (PPP) model. The NLO coefficients in terms of these correction vectors are given by

$$
\begin{aligned}
\alpha_{i j}(\omega)= & \left(\left\langle\phi_{i}^{(1)}(\omega)\left|\hat{\mu}_{j}\right| G\right\rangle+\left\langle\phi_{i}^{(1)}(-\omega)\left|\hat{\mu}_{j}\right| G\right\rangle\right), \\
\beta_{i j k}\left(\omega_{1}, \omega_{2}\right)= & \hat{P}_{i j k}\left(\left\langle\phi_{i}^{(1)}\left(-\omega_{1}-\omega_{2}\right)\left|\hat{\mu}_{j}\right| \phi_{k}^{(1)}\left(-\omega_{2}\right)\right\rangle\right), \\
\gamma_{i j k l}\left(\omega_{1}, \omega_{2}, \omega_{3}\right)= & \hat{P}_{i j k l}\left(\left\langle\phi_{i}^{(1)}\left(-\omega_{1}-\omega_{2}-\omega_{3}\right)\left|\hat{\mu}_{j}\right|\right.\right. \\
& \left.\left.\times \phi_{k l}^{(2)}\left(-\omega_{1}-\omega_{2},-\omega_{1}\right)\right\rangle\right),
\end{aligned}
$$

where the operators $\hat{P}_{i j k}$ and $\hat{P}_{i j k l}$ generate all permutations: $\left(-\omega_{\sigma}, i\right),\left(\omega_{1}, j\right),\left(\omega_{2}, k\right)$ and $\left(-\omega_{\sigma}, i\right),\left(\omega_{1}, j\right),\left(\omega_{2}, k\right)$, $\left(\omega_{3}, l\right)$ leading to six terms for $\beta$ (with $\omega_{\sigma}=\omega_{1}+\omega_{2}$ ) and 24 terms for $\gamma$ (with $\omega_{\sigma}=\omega_{1}+\omega_{2}+\omega_{3}$ ) respectively.

The $\phi_{i}$ 's so computed are exact within the Hilbert space chosen for the Hamiltonian. This is because the correction vectors implicitly incorporate all the excited states of the Hamiltonian in the configuration space in which it is defined. The linear algebraic equations that result can be solved efficiently by a small matrix algorithm developed by Ramasesha [28]. We report only the spatially averaged values of first-, second- and thirdorder NLO coefficients. The expressions for these are given by

$$
\begin{aligned}
\alpha_{\mathrm{av}} & =\sum_{i=1}^{3} \frac{1}{3} \alpha_{i i}, \\
\beta_{\mathrm{av}, i} & =\sum_{j=1}^{3} \frac{1}{3}\left(\beta_{i j j}+\beta_{j j i}+\beta_{j i j}\right), \\
\gamma_{\mathrm{av}} & =\sum_{i, j=1}^{3} \frac{1}{15}\left(2 \gamma_{i j j j}+\gamma_{i j j i}\right),
\end{aligned}
$$

where $i, j$ are Cartesian indices $(x, y, z)$.

The imaginary part of $\gamma$ gives the two-photon absorption coefficient. The cross-section of the twophoton absorption (TPA) coefficient is given by

$$
\delta_{\mathrm{tpa}}(\omega)=\frac{8 \pi^{2} \hbar \omega^{2}}{n^{2} c^{2}} \operatorname{Im} \gamma(-\omega ; \omega, \omega,-\omega) .
$$

Using a sum-over-states expression and assuming that the major contribution to TPA comes from the resonant terms, one can write the two-photon matrix elements as [29]

$$
T_{x, y}=\sum_{i} \frac{\left\langle g\left|\mu_{x}\right| i\right\rangle\left\langle i\left|\mu_{y}\right| f\right\rangle}{\omega_{i}-\omega_{f} / 2}+\frac{\left\langle g\left|\mu_{y}\right| i\right\rangle\left\langle i\left|\mu_{x}\right| f\right\rangle}{\omega_{i}-\omega_{f} / 2},
$$

where $\omega_{i}$ is the excitation energy of $i$ th state.
For linearly polarized monochromatic light, the orientational average of two-photon absorption crosssection has the property

$$
\delta_{\mathrm{tpa}} \propto \sum_{i, j}\left[2 T_{i i} T_{j j}+4 T_{i j} T_{j i}^{*}\right]
$$

where the summation $i, j$ runs over all three Cartesian coordinates. Using the first-order correction vector, we have computed the quantities $T_{i j}$ and then two-photon matrix elements within the ZINDO package. In our studies, we have computed the right-hand side of equation (13) and have called the quantity $\delta_{\text {tpa. }}$. Since our interest is in comparing different systems rather than obtaining absolute values, setting the proportionality constant to unity will not affect our analysis.

\section{Results and discussion}

\subsection{Molecular structures and geometry optimization}

We have used AM1 optimization without any symmetry constraint [30], available in the Gaussian package [31] to obtain the equilibrium geometry of the molecules. The structures of molecules studied in this work are shown in figure 1 . All the molecules have a conjugated backbone made up of stilbene or azobenzene, with a fixed acceptor group, which is a nitro group, $\mathrm{NO}_{2}$. The donor groups we have used are azaphosphanes and substituted azaphosphanes namely, $\left(\mathrm{R}_{1} \mathrm{R}_{2} \mathrm{R}_{3}\right) \mathrm{P}=\mathrm{N}$, $\mathrm{R}_{1}=\mathrm{R}_{2}=\mathrm{R}_{3}=\mathrm{H}, \mathrm{Me}, \mathrm{Ph}, \mathrm{NH}_{2}$ as well as $\mathrm{R}_{1}=\mathrm{R}_{2}=\mathrm{Ph}$, $\mathrm{R}_{3}=\mathrm{H}$ and $\mathrm{R}_{1}=\mathrm{Ph}, \mathrm{R}_{2}=\mathrm{R}_{3}=\mathrm{H}$. To vary the strength of the donor, we have varied the substituents at the phosphorus centre in azaphosphanes. Additionally, we have studied molecules based on stilbene as well as azobenzene to compare the effects of homo and hetero conjugations in the backbone.

The generic structure of the molecules studied is shown in figure 2 , where $\mathrm{X}_{1}=\mathrm{X}_{2}=\mathrm{C}$ corresponds to stilbenes and $\mathrm{X}_{1}=\mathrm{X}_{2}=\mathrm{N}$ corresponds to azobenzene. In tables 1 and 2 we have given the bond lengths of the prominent bonds while in tables 3 and 4 we have given the bond angles. We observe several interesting features from geometry optimization. The first point to be noted from tables 1 and 2 is that ring A (figure 2) has slight quinonoid structure with two different ring bond lengths $1.38 \pm 0.01$ and $1.42 \pm 0.01 \AA$, in all the molecules (i.e. in both stilbene and azobenzene derivatives), while ring $\mathrm{B}$ is benzenoid with all ring bond lengths being $1.40 \pm 0.01 \AA$. This can be due to the electron donation from the nitrogen atom in the azaphosphane group which leads to the quinonoid form of benzene ring A. However, there is no quinonoidal resonance structure involving ring $\mathrm{B}$ due to the electron 


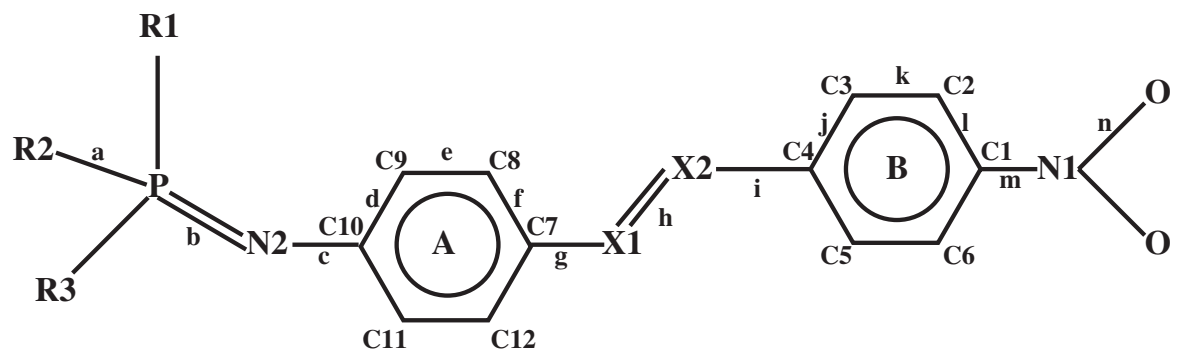

Figure 2. Generic structure of the molecules studied, with bond length and bond angle labels referred to in tables 1 to 4 .

Table 1. Optimized bond lengths $(\AA)$ for prominent bonds for stilbene derivatives.

\begin{tabular}{lcccccc}
\hline & \multicolumn{6}{c}{ Molecule } \\
\cline { 2 - 7 } Bond & I & II & III & IV & V & VI \\
\hline$a$ & 1.70 & 1.78 & 1.38 & 1.75 & 1.75 & 1.76 \\
$b$ & 1.69 & 1.66 & 1.67 & 1.66 & 1.66 & 1.66 \\
$c$ & 1.36 & 1.36 & 1.36 & 1.36 & 1.36 & 1.36 \\
$d$ & 1.43 & 1.44 & 1.42 & 1.42 & 1.42 & 1.44 \\
$e$ & 1.38 & 1.38 & 1.38 & 1.38 & 1.38 & 1.38 \\
$f$ & 1.41 & 1.41 & 1.40 & 1.40 & 1.41 & 1.41 \\
$g$ & 1.45 & 1.44 & 1.44 & 1.44 & 1.44 & 1.44 \\
$h$ & 1.35 & 1.35 & 1.35 & 1.35 & 1.35 & 1.35 \\
$i$ & 1.45 & 1.45 & 1.45 & 1.45 & 1.45 & 1.45 \\
$l$ & 1.40 & 1.40 & 1.40 & 1.40 & 1.40 & 1.40 \\
$m$ & 1.48 & 1.48 & 1.48 & 1.48 & 1.48 & 1.48 \\
$n$ & 1.21 & 1.21 & 1.21 & 1.21 & 1.21 & 1.21 \\
\hline
\end{tabular}

$h=1.33 \AA$ from experimental results [32] and $1.35 \AA$ from theoretical results [33].

Table 2. Optimized bond lengths $(\AA)$ for prominent bonds for azobenzene derivatives.

\begin{tabular}{lcccccc}
\hline & \multicolumn{5}{c}{ Molecule } \\
\cline { 2 - 7 } Bond & VII & VIII & IX & X & XI & XII \\
\hline$a$ & 1.70 & 1.78 & 1.38 & 1.74 & 1.75 & 1.76 \\
$b$ & 1.69 & 1.66 & 1.67 & 1.66 & 1.66 & 1.66 \\
$c$ & 1.36 & 1.35 & 1.35 & 1.35 & 1.35 & 1.35 \\
$d$ & 1.42 & 1.44 & 1.43 & 1.43 & 1.43 & 1.44 \\
$e$ & 1.38 & 1.37 & 1.38 & 1.38 & 1.38 & 1.39 \\
$f$ & 1.41 & 1.42 & 1.41 & 1.41 & 1.41 & 1.41 \\
$g$ & 1.42 & 1.42 & 1.42 & 1.42 & 1.42 & 1.42 \\
$h$ & 1.23 & 1.24 & 1.23 & 1.23 & 1.23 & 1.23 \\
$i$ & 1.44 & 1.44 & 1.44 & 1.44 & 1.44 & 1.44 \\
$l$ & 1.40 & 1.40 & 1.40 & 1.40 & 1.40 & 1.40 \\
$m$ & 1.48 & 1.48 & 1.48 & 1.48 & 1.48 & 1.48 \\
$n$ & 1.21 & 1.21 & 1.21 & 1.21 & 1.21 & 1.21 \\
\hline$h$ & & & & & & \\
& $1.26 \mathrm{~A}$ & & & &
\end{tabular}

$h=1.26 \AA$ from another theoretical calculations [33] and $h=1.25 \AA$ from experimental studies [32]. $i=1.46 \AA$ from another theoretical calculations [34].
Table 3. Optimized bond angles in degrees for prominent bonds for stilbene derivatives.

\begin{tabular}{lcccccc}
\hline & \multicolumn{5}{c}{ Molecule } \\
\cline { 2 - 7 } Bond angle & I & II & III & IV & V & VI \\
\hline$\angle \mathrm{R}_{1} \mathrm{PN}_{2}$ & 115.1 & 117.2 & 116.9 & 118.2 & 118.0 & 116.0 \\
$\angle \mathrm{R}_{2} \mathrm{PN}_{2}$ & 115.0 & 115.4 & 116.9 & 117.6 & 115.5 & 115.6 \\
$\angle \mathrm{R}_{3} \mathrm{PN}_{2}$ & 107.9 & 105.5 & 105.6 & 105.4 & 108.6 & 105.9 \\
$\angle \mathrm{PN}_{2} \mathrm{C}_{10}$ & 119.3 & 119.1 & 116.1 & 117.7 & 117.2 & 120.7 \\
$\angle \mathrm{N}_{2} \mathrm{C}_{10} \mathrm{C}_{9}$ & 127.0 & 126.8 & 126.0 & 126.2 & 126.5 & 127.0 \\
$\angle \mathrm{N}_{2} \mathrm{C}_{10} \mathrm{C}_{11}$ & 116.6 & 117.1 & 117.6 & 117.4 & 117.2 & 116.8 \\
$\angle \mathrm{C}_{11} \mathrm{C}_{10} \mathrm{C}_{9}$ & 116.4 & 116.1 & 116.4 & 116.4 & 116.3 & 116.2 \\
$\angle \mathrm{C}_{9} \mathrm{C}_{8} \mathrm{C}_{7}$ & 121.8 & 121.9 & 121.4 & 121.4 & 121.5 & 121.9 \\
$\angle \mathrm{C}_{8} \mathrm{C}_{7} \mathrm{X}_{2}$ & 119.2 & 119.2 & 123.2 & 123.2 & 123.2 & 119.2 \\
$\angle \mathrm{C}_{7} \mathrm{X}_{2} \mathrm{X}_{1}$ & 125.5 & 125.7 & 125.6 & 125.6 & 125.6 & 125.7 \\
$\angle \mathrm{X}_{2} \mathrm{X}_{1} \mathrm{C}_{4}$ & 124.5 & 124.7 & 124.6 & 124.6 & 124.7 & 124.7 \\
$\angle \mathrm{X}_{1} \mathrm{C}_{4} \mathrm{C}_{3}$ & 118.9 & 118.9 & 118.9 & 118.9 & 118.9 & 118.9 \\
$\angle \mathrm{X}_{1} \mathrm{C}_{4} \mathrm{C}_{5}$ & 122.3 & 122.5 & 122.4 & 122.4 & 122.5 & 122.5 \\
$\angle \mathrm{C}_{1} \mathrm{~N}_{101}$ & 119.7 & 119.8 & 119.1 & 119.1 & 119.1 & 119.3 \\
\hline
\end{tabular}

Table 4. Optimized bond angles in degrees for prominent bonds for azobenzene derivatives.

\begin{tabular}{lcccccc}
\hline & \multicolumn{5}{c}{ Molecule } \\
\cline { 2 - 7 } Bond angle & VII & VIII & IX & $\mathrm{X}$ & $\mathrm{XI}$ & $\mathrm{XII}$ \\
\hline$\angle \mathrm{R}_{1} \mathrm{PN}_{2}$ & 115.0 & 115.9 & 116.6 & 116.0 & 115.4 & 115.8 \\
$\angle \mathrm{R}_{2} \mathrm{PN}_{2}$ & 115.0 & 115.9 & 116.6 & 116.0 & 117.8 & 115.5 \\
$\angle \mathrm{R}_{3} \mathrm{PN}_{2}$ & 107.8 & 105.3 & 105.5 & 110.3 & 108.5 & 105.9 \\
$\angle \mathrm{PN}_{2} \mathrm{C}_{10}$ & 119.5 & 119.2 & 116.2 & 115.5 & 117.4 & 120.8 \\
$\angle \mathrm{N}_{2} \mathrm{C}_{10} \mathrm{C}_{9}$ & 126.9 & 126.8 & 125.9 & 126.2 & 126.5 & 127.0 \\
$\angle \mathrm{N}_{2} \mathrm{C}_{10} \mathrm{C}_{11}$ & 116.7 & 117.1 & 117.7 & 117.6 & 117.3 & 116.9 \\
$\angle \mathrm{C}_{11} \mathrm{C}_{10} \mathrm{C}_{9}$ & 116.3 & 116.1 & 116.4 & 116.3 & 116.3 & 116.1 \\
$\angle \mathrm{C}_{9} \mathrm{C}_{8} \mathrm{C}_{7}$ & 121.2 & 121.4 & 121.1 & 121.2 & 121.21 & 121.4 \\
$\angle \mathrm{C}_{8} \mathrm{C}_{7} \mathrm{X}_{2}$ & 125.9 & 125.9 & 125.8 & 125.9 & 125.9 & 125.9 \\
$\angle \mathrm{C}_{7} \mathrm{X}_{2} \mathrm{X}_{1}$ & 120.5 & 120.6 & 120.5 & 120.6 & 120.6 & 120.6 \\
$\angle \mathrm{X}_{2} \mathrm{X}_{1} \mathrm{C}_{4}$ & 118.8 & 118.9 & 118.7 & 118.7 & 118.7 & 119.0 \\
$\angle \mathrm{X}_{1} \mathrm{C}_{4} \mathrm{C}_{3}$ & 123.9 & 123.9 & 123.9 & 123.9 & 123.9 & 124.0 \\
$\angle \mathrm{X}_{1} \mathrm{C}_{4} \mathrm{C}_{5}$ & 116.9 & 116.9 & 116.9 & 117.0 & 117.0 & 116.9 \\
$\angle \mathrm{C}_{1} \mathrm{~N}_{1} \mathrm{O}_{1}$ & 119.0 & 119.1 & 119.1 & 119.1 & 119.1 & 119.1 \\
\hline
\end{tabular}


withdrawing nature of $\mathrm{NO}_{2}$. Thus ring $\mathrm{B}$ will have a benzenoid form. However, experimental structural parameters are not known in the literature for comparison with our theoretical values.

The $\mathrm{C}-\mathrm{N}$ bond associated with ring $\mathrm{A}$ is $1.355 \pm$ $0.005 \AA$ and that with ring $\mathrm{B}$ is $1.48 \AA$. The $\mathrm{N}-\mathrm{O}$ bond length is $1.21 \AA$. All three bond lengths are insensitive to substitution. Geometry optimization of nitrosubstituted azobenzene and stilbene by the AM1 method gives the bond length of $\mathrm{N}-\mathrm{O}$ to be $1.20 \AA$ [35]. The $\mathrm{P}-\mathrm{N}$ bond length, however, depends upon substitution and is the same in both azobenzene and stilbene derivatives. The $\mathrm{P}-\mathrm{N}$ bond length is maximum for amino substitution $1.69 \AA$, it is $1.67 \AA$ for hydrogen and $1.66 \AA$ for all the other substitutions. The $\mathrm{P}-\mathrm{N}$ bond length known in the literature for linear chains is $1.62 \AA$ [36].

The $\mathrm{NO}_{2}$ group is in the same plane as ring $\mathrm{B}$. Ring $\mathrm{B}$ makes an angle of $18^{\circ}$ and $33^{\circ}$ with ring $\mathrm{A}$, in stilbene and azobenzene derivatives respectively. The $\mathrm{P}-\mathrm{N}$ bond and one of the substituents, $R_{1}$, is nearly in-plane with ring $A$, when all three susbstituents are the same. As the susbstituent becomes bulkier, this coplanarity is lost. The substituents at the phosphorus site in I to XII are, in general, not in the plane of ring $\mathrm{A}$, while for unsubstituted and methyl substituted systems, one of the substituents is in the same plane as ring $\mathrm{A}$, in all other cases none of the susbstituent lie in the plane of ring $\mathrm{A}$.

We have used these optimized geometries to compute excitation energies and optical properties, within the ZINDO package [25]. In order to fix the number of single and double excitations, we have varied the number of HOMOs (highest occupied molecular orbitals) and LUMOs (lowest unoccupied molecular orbitals) involved in these excitations. For each molecule, we have experimented with Rumer CI calculations using 10 and 11 HOMOs and an equal number of LUMOs for single excitations. For double excitations, we have used 3 to 6 HOMOs and 3 to 6 LUMOs. We have computed the lowest five singlet excitations in each case. Variation of these excitations as a function of the level of CI is shown in figure 3 for all twelve cases. We find that using 11 HOMOs and 11 LUMOs for single excitations together with 6 HOMOs and 6 LUMOs for double excitations gives almost uniformly converged energies for all the lowest five states. The Hilbert space of singlets spanned by these is 788 Rumer configurations. In all our calculations we have used 447 configurations (11 singlets and 5 doublets) and calculated the ground and excited state properties.

The ground state dipole moment of all the stilbene and azobenzene derivatives are shown in figure 4 . The azobenzene derivatives have a slightly larger dipole moment than the corresponding stilbene derivatives.
We note that the amino derivative has the smallest dipole while the triphenyl derivative has the largest dipole. The dipole moment of the phenyl derivatives are nearly the same, independent of the number of phenyl groups in the molecule. In order to understand these trends a little more in detail, we have computed the charge densities.

The charge densities at phosphorus and nitrogen of the azaphosphane group are presented in table 5 . The maximum electron deficiency is at the $\mathrm{P}$ centre when the substituent is an amino group while it is minimum in the unsubstituted case. Replacement of each hydrogen atom by a phenyl group causes nearly 0.04 electrons to transfer from $\mathrm{P}$, while the change in charge on the $\mathrm{N}$ connected to $\mathrm{P}$ is insignificant $(\leq 0.01)$. These charge densities only depend on the susbstitution and are the same for corresponding derivatives of stilbenes and azobenzenes. Again, for the amino group, the charge on $\mathrm{N}$ is maximum. The electrons are withdrawn from $\mathrm{P}$, whereas electrons are added to $\mathrm{N}$. The magnitude of the electronic charge withdrawn is almost the same for phenyl and methyl groups. This is reflected in the bondlength variation also. The bond length between $\mathrm{R}$ and $\mathrm{P}$, the bond connecting the substituent and the $\mathrm{P}$ atoms, are almost of same magnitude (1.76 and $1.78 \AA$ ). The formal charge on $\mathrm{P}$ is independent of the backbone. This confirms the earlier prediction that the polarization is effective only in the immediate neighbourhood of the substituent [37]. It is also interesting to note that the dipole moment is larger for the molecules in which charge separation between $\mathrm{P}$ and $\mathrm{N}$ is smaller. This can be understood from the fact that these molecules are also larger and small charge separated by larger distances results in larger dipole moments.

\section{Linear optical properties}

In table 6, we present the singlet excitation energies and transition dipole moments for stilbene and azobenzene derivatives for different substituents for the lowest three excitations. The lowest optical gap for stilbene derivatives is always higher than that for the corresponding azobenzene derivatives. It is maximum for amino substitution in both cases. While the optical gap of the phenyl derivatives decreases as we increase the number of phenyl groups for the case of stilbene derivatives, the same shows an increasing trend for azobenzene derivatives. Experimental studies on nitro-stilbene derivatives place the optical gap in the range $3.0 \pm 0.2 \mathrm{eV}$ for different substituents like $-\mathrm{Br}, \mathrm{OCH}_{3}, \mathrm{~N}\left(\mathrm{CH}_{3}\right)_{2}$, and $\mathrm{CH}_{3} \mathrm{~S}$ at the para position [37, 38]. However, what is interesting is that the lowest excitation in stilbene 


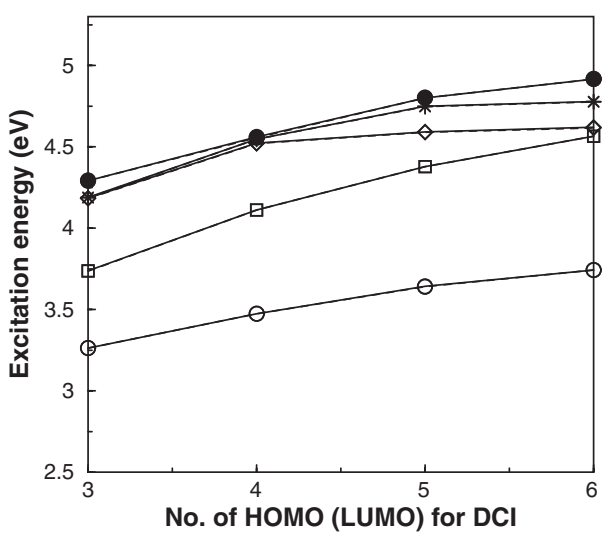

III

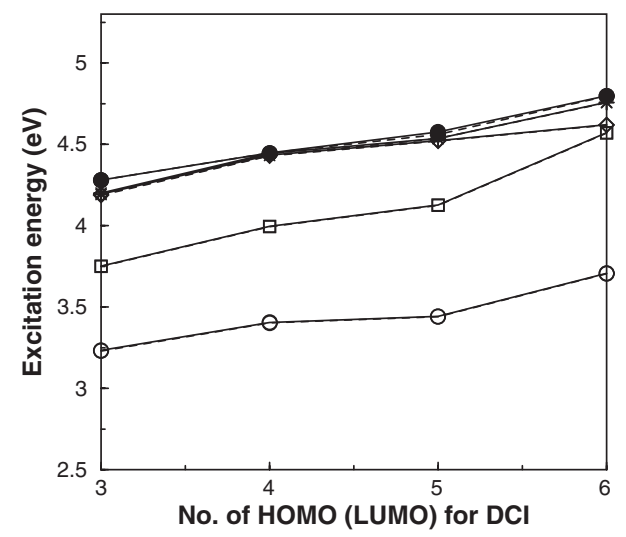

v

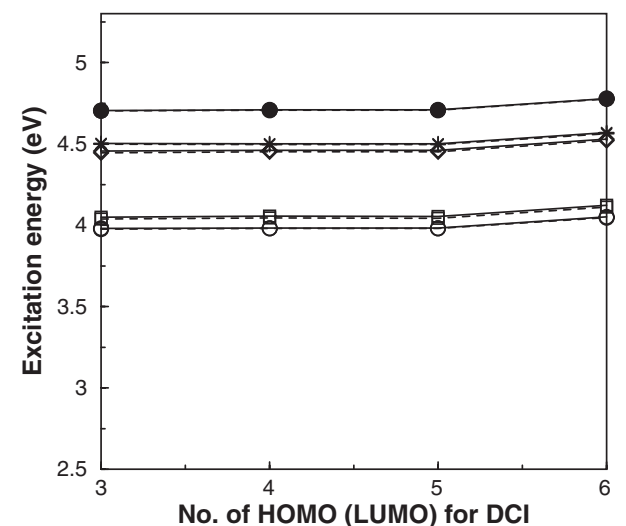

II

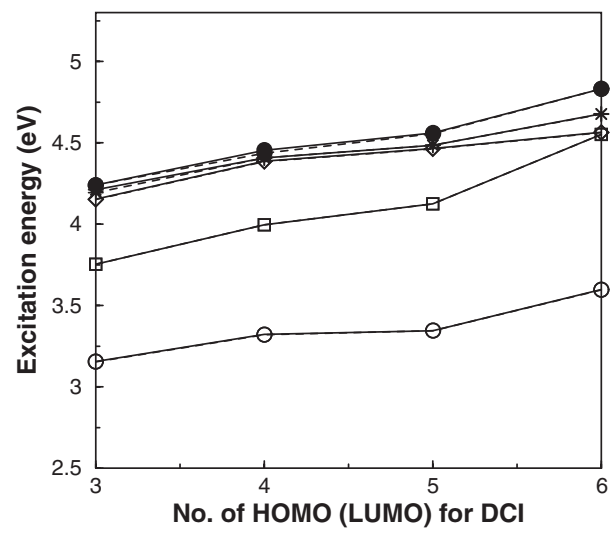

IV

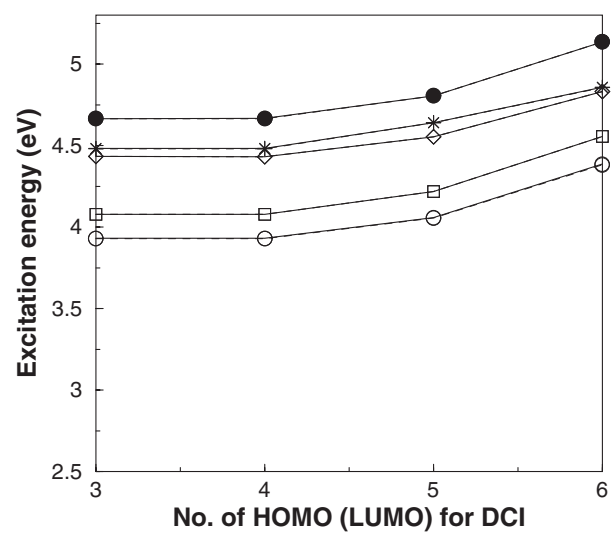

VI

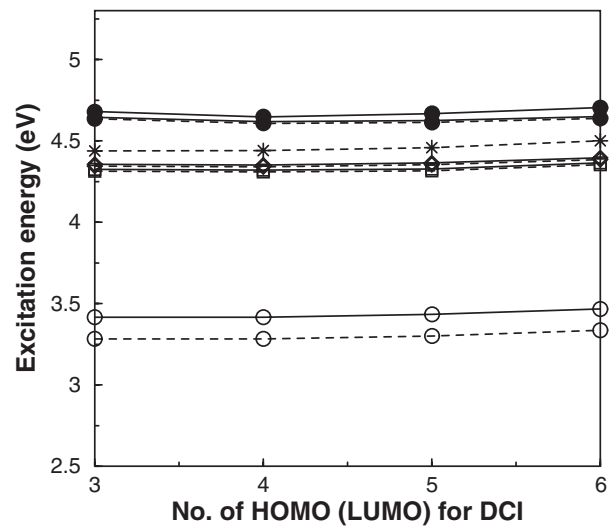

Figure 3. Dependence of energy of state $n$ (for $n=1(\circ), 2(\square), 3(\diamond), 4(*), 5(\bullet))$ on the level of the SDCI calculation in ZINDO. For each state of every molecule, two different numbers of HOMOs and LUMOs for single excitation are considered. Solid lines correspond to 10 HOMOs and 10 LUMOs involved in the single CI excitations, while the dotted lines correspond to 11 HOMOs and 11 LUMOs. For each of these cases doubly excited configurations from 3 HOMOs and 3 LUMOs to 6 HOMOs and 6 LUMOs are considered and are shown as an $x$ axis variable.

derivatives is optically intense while that in azobenzene derivatives is very weak, almost optically forbidden.

In figure 5 we have shown the one-photon absorption cross-section for the allowed excited state for stilbene and azobenzene derivatives. If we look at the second lowest optical excitations in these systems, for the stilbene derivatives, the second excitation is forbidden, while for azobenzene derivatives it is intense. The third optical excitation is nearly forbidden in both cases. This trend was also observed in earlier studies of 


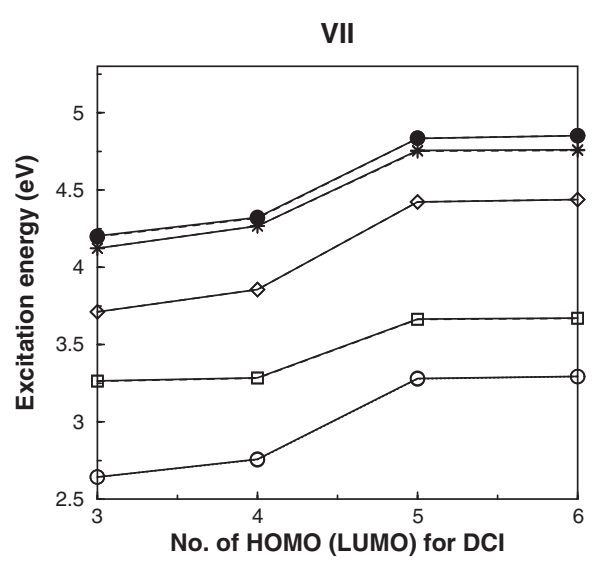

IX

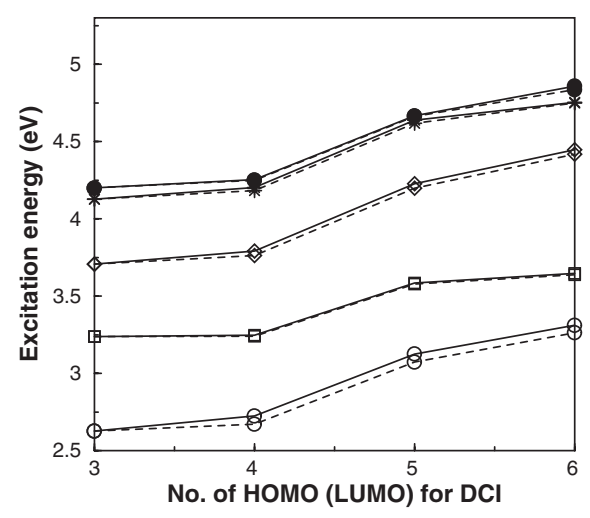

$\mathrm{XI}$

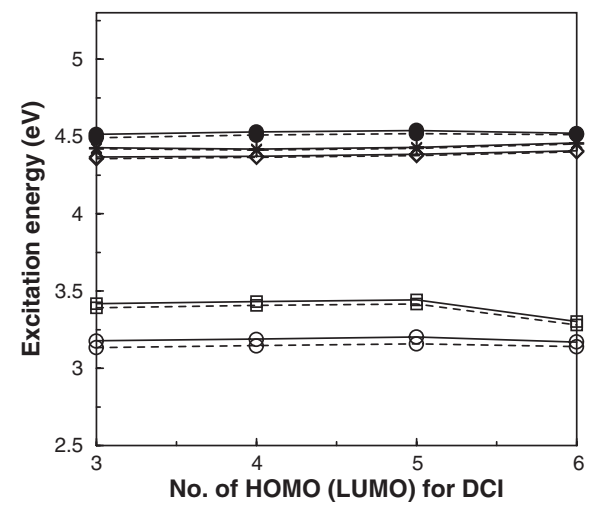

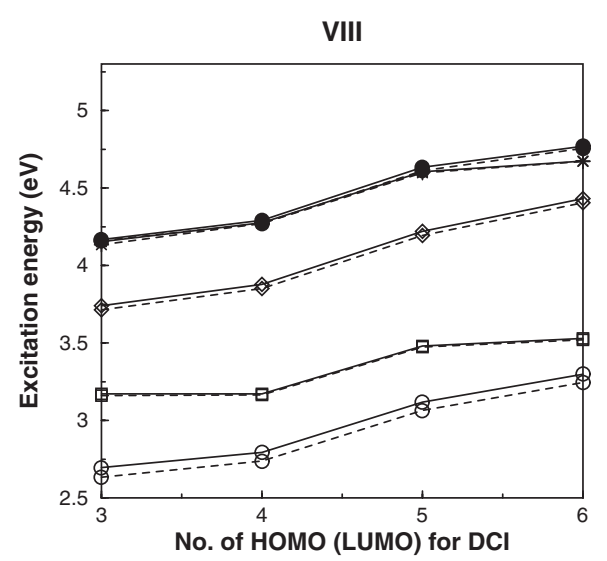

$\mathrm{x}$

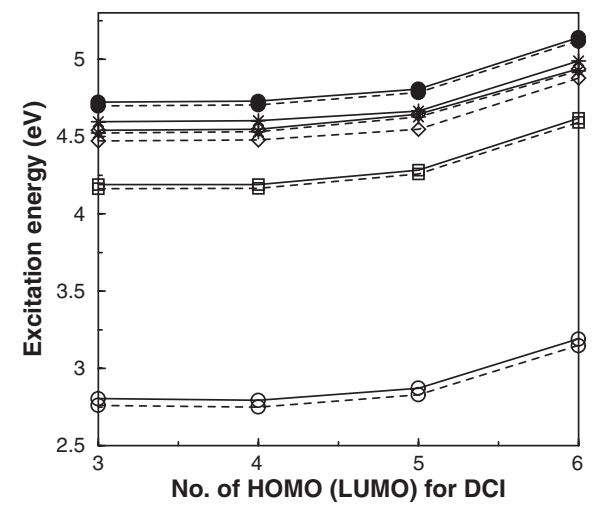

XII

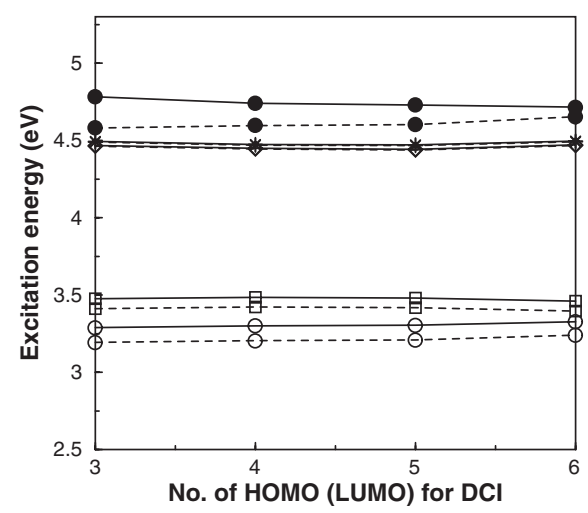

Figure 3. Continued.

Chen et al. [33]. The observation of an optically 'forbidden' state below the optically 'allowed' state in the azobenzene system leads us to conclude that the role of electron correlations is stronger in the case of azobenzene systems than in the stilbene systems. This could be attributed to the fact that the central double bond connecting the two benzene rings in azobenzene breaks the electron conjugation. This is expected with heteroatoms as they can act as electron trapping or repelling sites due to different orbital (or site) energies and correlation strengths.
The role of substituents in altering the optical gap to the strongly allowed state is similar in both stilbene and azobenzene derivatives. The larger optical gap in azobenzene derivatives compared to stilbene derivatives can be attributed to the restricted delocalization in the azobenzene systems. Similar magnitudes of oscillator strengths for the strongly allowed excitations in the azobenzene and stilbene systems also implies very similar charge distributions in the corresponding lowlying states of the system. Introducing phenyl groups on the phosphorus site marginally reduces the optical gaps, 


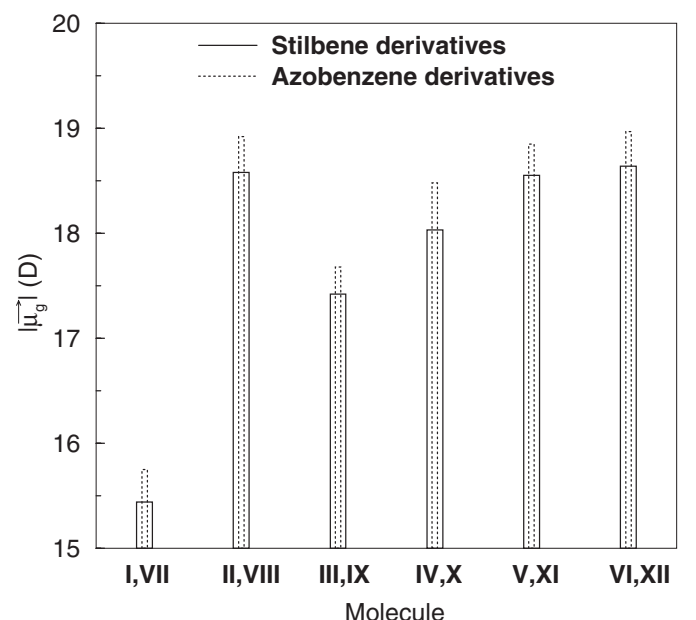

Figure 4. Ground state dipole moment of azobenzene and stilbene derivatives.

Table 5. Mulliken charges on phosphorus and azaphosphane nitrogen (connected to the phosphorus atom)for different substituents using Mulliken population analysis. The charge on the phosphorus site is +ve while on the nitrogen site it is $-v e$, corresponding to deficient and excess electron density at these sites.

\begin{tabular}{lcc}
\hline Molecule & $\mathrm{P}$ & $\mathrm{N}$ \\
\hline I & 1.29 & -0.85 \\
II & 0.76 & -0.81 \\
III & 0.66 & -0.79 \\
IV & 0.70 & -0.80 \\
V & 0.74 & -0.81 \\
VI & 0.78 & -0.82 \\
VII & 1.29 & -0.84 \\
VIII & 0.76 & -0.81 \\
IX & 0.66 & -0.78 \\
X & 0.70 & -0.79 \\
XI & 0.74 & -0.80 \\
XII & 0.78 & -0.81 \\
\hline
\end{tabular}

as expected, due to increased conjugation length of the molecule.

\section{Linear and nonlinear optical response coefficients}

We have computed the linear polarizability, the second harmonic generation coefficient (SHG), the electric field induced second harmonic generation coefficient (EFISH), due to alignment of dipoles in a static electric field, and the third harmonic generation coefficient (THG). These quantities have been computed at two excitation frequencies, 0.65 and $1.17 \mathrm{eV}$. In what follows we discuss these results in detail.
Table 6. Optical gap(in eV), transition dipole moments (TD) (in D)and one-photon absorption (OPA) values(in au) for the lowest three excited states for stilbene and azobenzene derivatives.

\begin{tabular}{lccc}
\hline Molecule & Optical gap & TD & OPA \\
\hline I & 3.64 & 8.71 & 1.05 \\
& 4.38 & 0.14 & 0.00 \\
II & 4.59 & 1.75 & 0.05 \\
& 3.34 & 9.29 & 1.10 \\
& 4.12 & 0.15 & 0.00 \\
III & 4.46 & 1.89 & 0.06 \\
& 3.44 & 9.16 & 1.10 \\
& 4.12 & 0.16 & 0.00 \\
IV & 4.52 & 1.69 & 0.05 \\
& 3.40 & 9.35 & 1.13 \\
& 4.04 & 0.12 & 0.00 \\
V & 4.45 & 0.27 & 0.00 \\
& 3.32 & 9.85 & 1.22 \\
& 3.79 & 0.04 & 0.00 \\
VI & 4.30 & 1.17 & 0.02 \\
& 3.30 & 10.19 & 1.30 \\
& 4.32 & 0.21 & 0.00 \\
VII & 4.35 & 0.32 & 0.00 \\
& 3.28 & 1.46 & 0.03 \\
& 3.66 & 8.12 & 0.92 \\
VIII & 4.42 & 0.08 & 0.00 \\
& 3.06 & 1.59 & 0.03 \\
& 3.47 & 8.84 & 1.03 \\
IX & 4.19 & 0.13 & 0.00 \\
& 3.07 & 1.35 & 0.02 \\
& 3.58 & 8.73 & 1.04 \\
X & 4.20 & 0.12 & 0.00 \\
& 3.00 & 1.81 & 0.04 \\
XI & 3.43 & 9.22 & 1.11 \\
& 4.04 & 0.15 & 0.00 \\
& 3.16 & 2.90 & 0.10 \\
& 3.42 & 8.90 & 1.03 \\
& 4.38 & 2.11 & 0.07 \\
& 3.21 & 2.77 & 0.09 \\
& 4.42 & 9.48 & 1.17 \\
& & 0.20 & 0.00 \\
\hline & & & \\
& & &
\end{tabular}

\subsection{Linear polarizability}

The orientationally averaged polarizability $\left(\alpha_{\text {av }}\right)$ of all the molecules studied by us is shown in figure 6 . The important point to note here is that the tumbling averaged value of $\alpha$ for all the substituents are marginally lower in the case of azobenzenes compared to stilbenes both at 0.65 and $1.17 \mathrm{eV}$ excitation frequencies. This is due to the slightly larger optical gaps and smaller transition dipole moments found in the azobenzene molecules compared to the stilbenes. The polarizability 


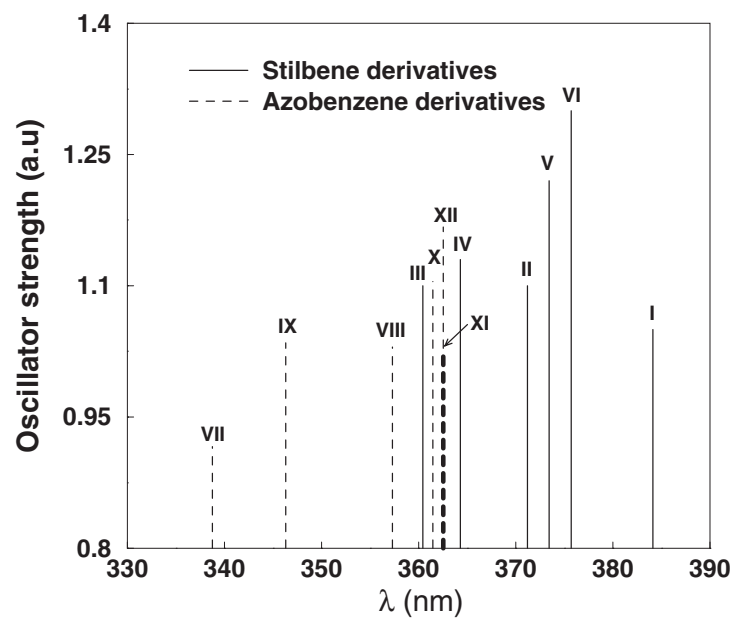

Figure 5. Oscillator strengths corresponding to $\lambda_{\max }$ for stilbene and azobenzene derivatives.
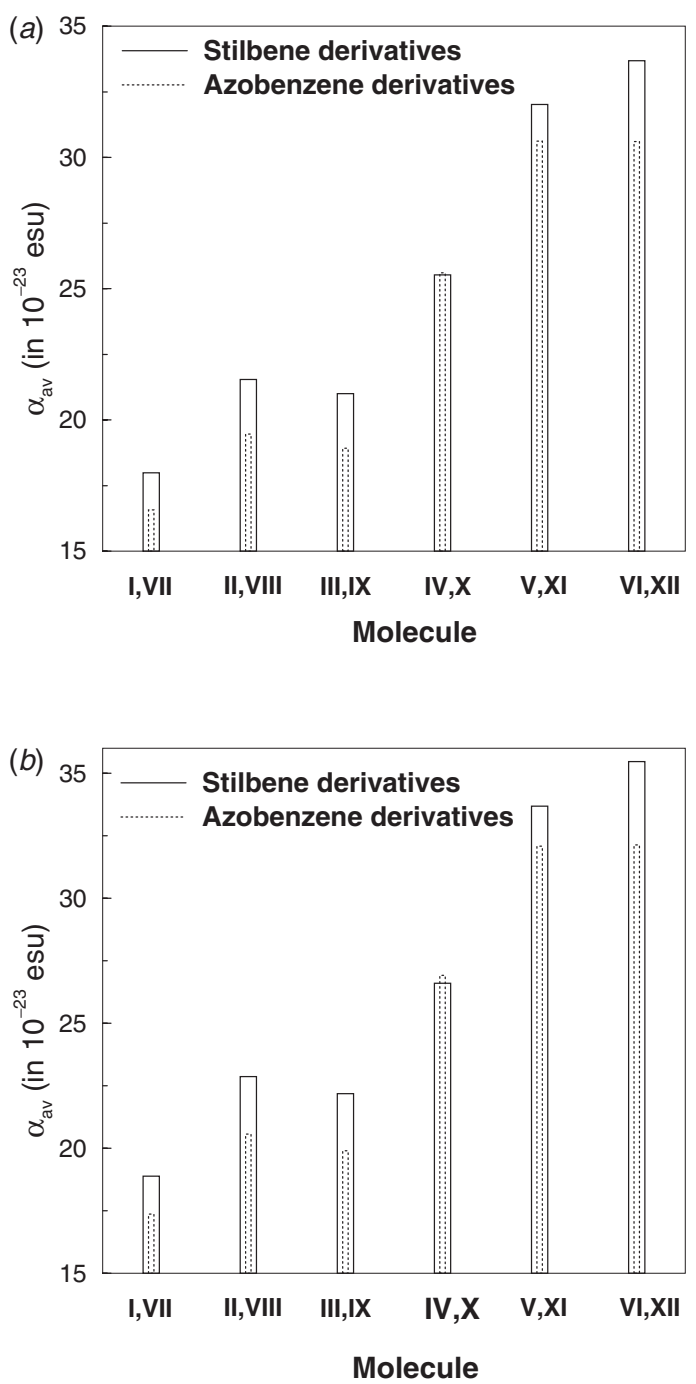

Figure 6. The average value of polarizability, $\alpha_{\mathrm{av}}$, for stilbene and azobenzene derivatives at $(a) 0.65 \mathrm{eV}$ and $(b) 1.17 \mathrm{eV}$. is very weakly dependent on frequency in this range since the optical gaps are all $\geq 3.3 \mathrm{eV}$. The polarizability is larger for systems with smaller optical gaps. In the case of molecules IV, V, VI and X, XI and XII, the polarizability increases with increasing number of phenyl groups. However, the increase in $\alpha_{\mathrm{av}}$ in going from two to three phenyl rings is only marginal. In the case of stilbene derivatives, the transition dipole moment to the first excited state increases from unsubstituted molecules to $\mathrm{Ph}_{3}$ substituted molecules, whereas the optical gap decreases. Hence the linear polarizability increases as $\mathrm{H}$ atoms are replaced by $\mathrm{Ph}$ groups. The transition dipole moment of the $\mathrm{CH}_{3}$ substituted molecule is larger than for the unsubstituted molecule and the optical gap has an opposite trend. Hence the $\mathrm{CH}_{3}$ substituted molecule has larger $\alpha$ than the unsubstituted molecule. For the $\mathrm{NH}_{2}$ substituted molecule both optical gap (largest) and transition dipole moment (smallest) are such as to result in smaller linear polarizability. A similar trend is observed for azobenzene derivatives.

\subsection{SHG, THG and EFISH studies}

In figure 7 , we show $\left|\beta_{\text {av }}\right|$, the magnitude of the tumbling averaged SHG coefficients $\beta$, for various molecules at 0.65 and $1.17 \mathrm{eV}$ excitation frequencies. We note that the azobenzene derivatives have smaller $\left|\beta_{\mathrm{av}}\right|$ values than stilbene derivatives at both frequencies, although the $\left|\beta_{\mathrm{av}}\right|$ values for both systems are larger at the excitation frequency of $1.17 \mathrm{eV}$ compared to that at $0.65 \mathrm{eV}$. The slight increase in $\left|\beta_{\text {av }}\right|$ at higher frequency is because the system is closer to the resonance condition at $1.17 \mathrm{eV}$ than at $0.65 \mathrm{eV}$. We also find that the trend in $\left|\beta_{\mathrm{av}}\right|$ is well explained by the Oudar-Chemla two-level formula, which involves excitation energy to the key charge transfer $(\mathrm{CT})$ state $\left(E_{\mathrm{ge}}\right)$, the difference in dipole moment between ground and CT state $(\Delta \mu)$ and the magnitude of transition dipole moment $\left(\mu_{\mathrm{ge}}^{2}\right)$. $\beta$ is given by $\beta=\mu_{\mathrm{ge}}^{2} \Delta \mu / E_{\mathrm{ge}}^{2}$. Marder and co-workers have shown that the variation of $\beta$ is similar to the change in dipole moment, $\Delta \mu$ [39]. In figure 8 we compare the $\beta_{\text {av }}$ values from the two-state model and our calculation. The trend in the evolution of $\beta$ is similar in both cases although the two-state model $\beta$ values are overestimated by a factor of 2 or more, as obtained from the correction vector method. From table 7, we see that the change in dipole moment is smallest for $\mathrm{NH}_{2}$ substitution. A large optical gap and a small transition dipole moment, together with a small $\Delta \mu$, leads to a small $\beta$ value. Even though, the change in the dipole moment is smaller for $\mathrm{HPh}_{2}$ and $\mathrm{Ph}_{3}$ substitutions, the small optical gap and the large transition dipole moment, as well as the large change 

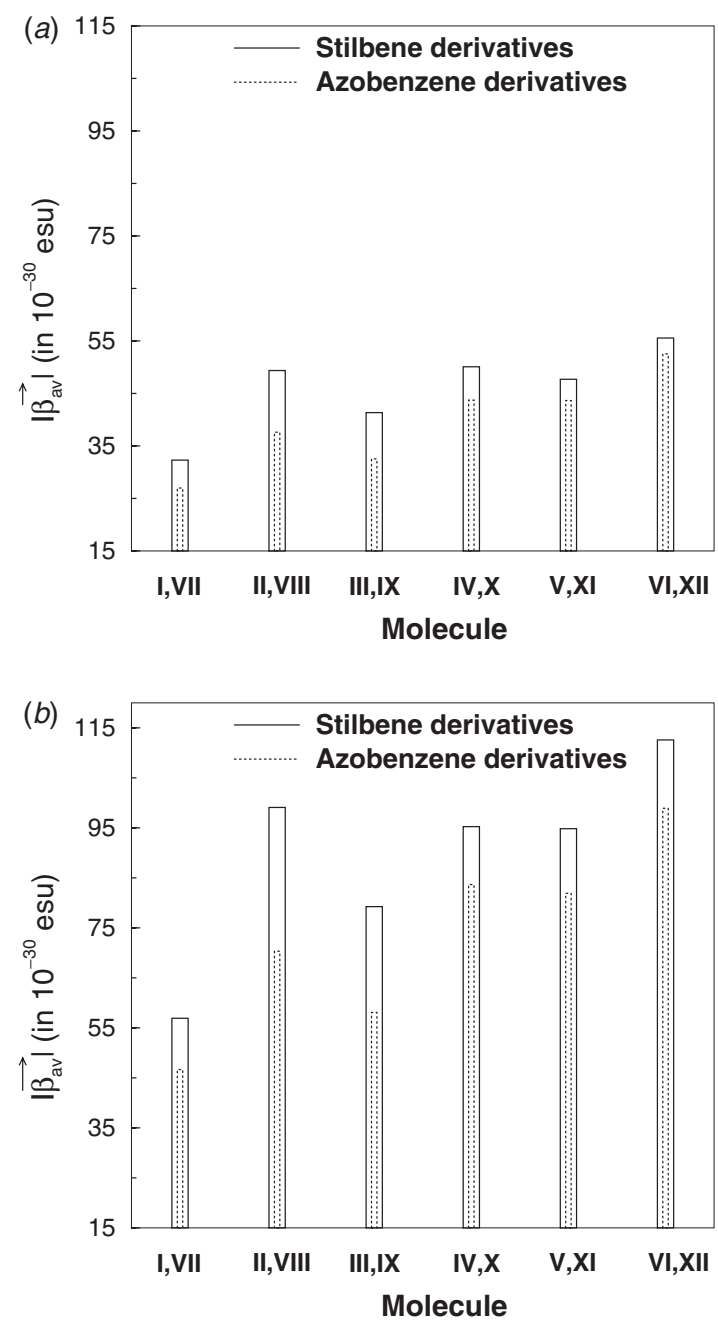

Figure 7. The average value of first-order hyperpolarizability $\left(\left|\beta_{\mathrm{av}}\right|\right)$ for stilbene and azobenzene derivatives at $(a) 0.65 \mathrm{eV}$ and $(b) 1.17 \mathrm{eV}$.

in the dipole moment to the third excited state, favour larger $\beta$ values for both $\mathrm{HPh}_{2}$ and $\mathrm{Ph}_{3}$ substitutions.

Romaniello and Lelj have obtained NLO coefficients from the PM3 method for fluorine substituted nitrostilbene derivatives [40]. The first-order hyperpolarizability for a $\mathrm{OCH}_{3}$ substituted nitro-stilbene derivative is $\approx 31.2$ (in $10^{-30} \mathrm{esu}$ ) and for the compound with one more phenyl linkage in between, it is 47.2 (in $10^{-30} \mathrm{esu}$ ). Our results are in agreement with their calculations. The $\left|\beta_{\mathrm{av}}\right|$ value is maximum for phenyl-substituted groups both for stilbene and azobenzene derivatives. Similar comparison by Ulman et al. between a series of biphenyl, stilbene and azobenzene derivatives using both SOS and finite field calculations shows that $\left|\beta_{\mathrm{av}}\right|$ values for both stilbene and azobenzene derivatives are very similar [41]. The values of $\left|\beta_{\mathrm{av}}\right|$ are $\approx 40 \times 10^{-30}$ esu at $\lambda=1907 \mathrm{~nm}(\hbar \omega=0.65 \mathrm{eV})$.
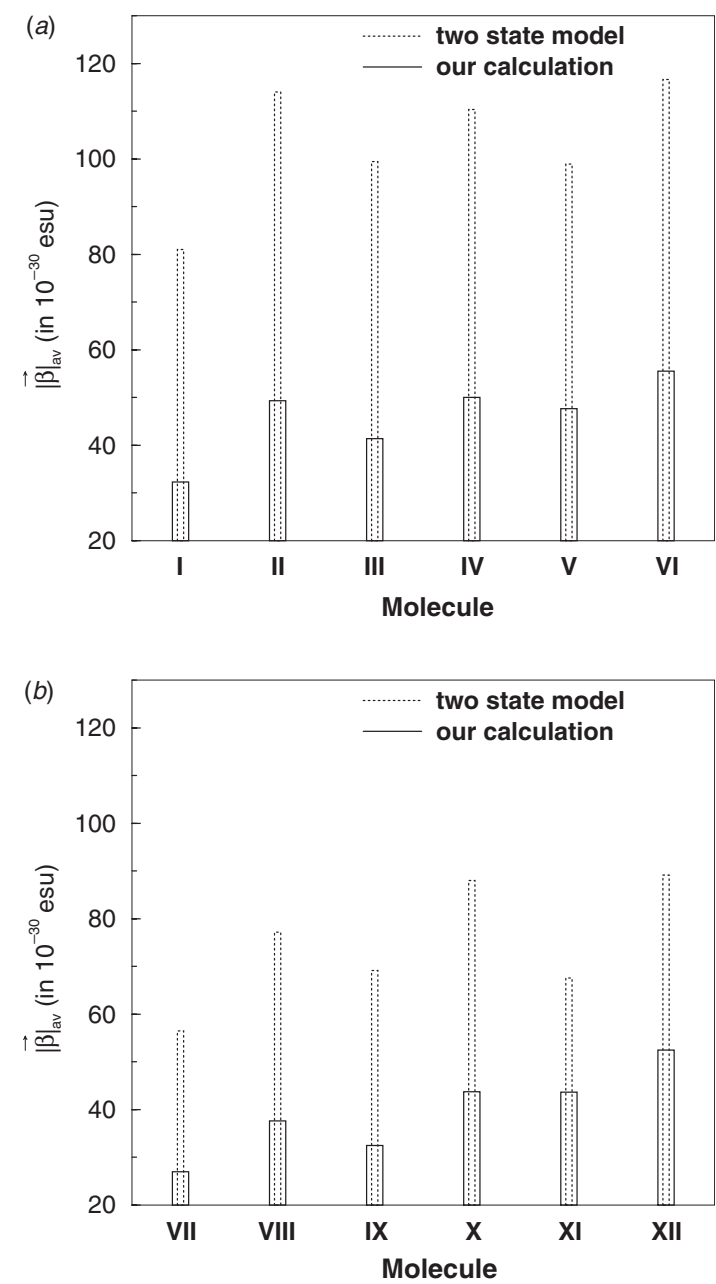

Figure 8. Comparison of the average value of first-order hyperpolarizability $\left(\left|\beta_{\text {av }}\right|\right)$ from the two-state model with results from the correction vector approach for $(a)$ stilbene and $(b)$ azobenzene derivatives at $0.65 \mathrm{eV}$.

Table 7. The ground state dipole moment ( $\mu_{\mathrm{g}}$ in D), difference in dipole moment between the ground and the first $\left(\Delta \mu_{1}\right.$ in D) and the second $\left(\Delta \mu_{2}\right.$ in D) excited states and EFISH values (in $10^{-48} \mathrm{esu}$ ) at $0.65 \mathrm{eV}$.

\begin{tabular}{lcrrr}
\hline Molecule & $\mu_{\mathrm{g}}$ & $\Delta \mu_{1}$ & $\Delta \mu_{2}$ & EFISH \\
\hline I & 15.44 & 14.15 & 2.59 & 472.84 \\
II & 18.58 & 14.74 & 2.83 & 847.09 \\
III & 17.42 & 14.02 & 2.77 & 653.66 \\
IV & 18.03 & 14.59 & 2.51 & 832.30 \\
V & 18.55 & 11.24 & 2.50 & 829.85 \\
VI & 18.64 & 12.23 & 21.50 & 957.52 \\
VII & 15.75 & 0.09 & 11.47 & 403.43 \\
VIII & 18.99 & 0.19 & 11.89 & 659.16 \\
IX & 17.68 & 0.27 & 11.63 & 521.55 \\
X & 18.48 & 0.17 & 12.18 & 750.35 \\
XI & 18.85 & 1.93 & 9.98 & 767.63 \\
XII & 18.97 & 2.11 & 11.60 & 917.29 \\
\hline
\end{tabular}



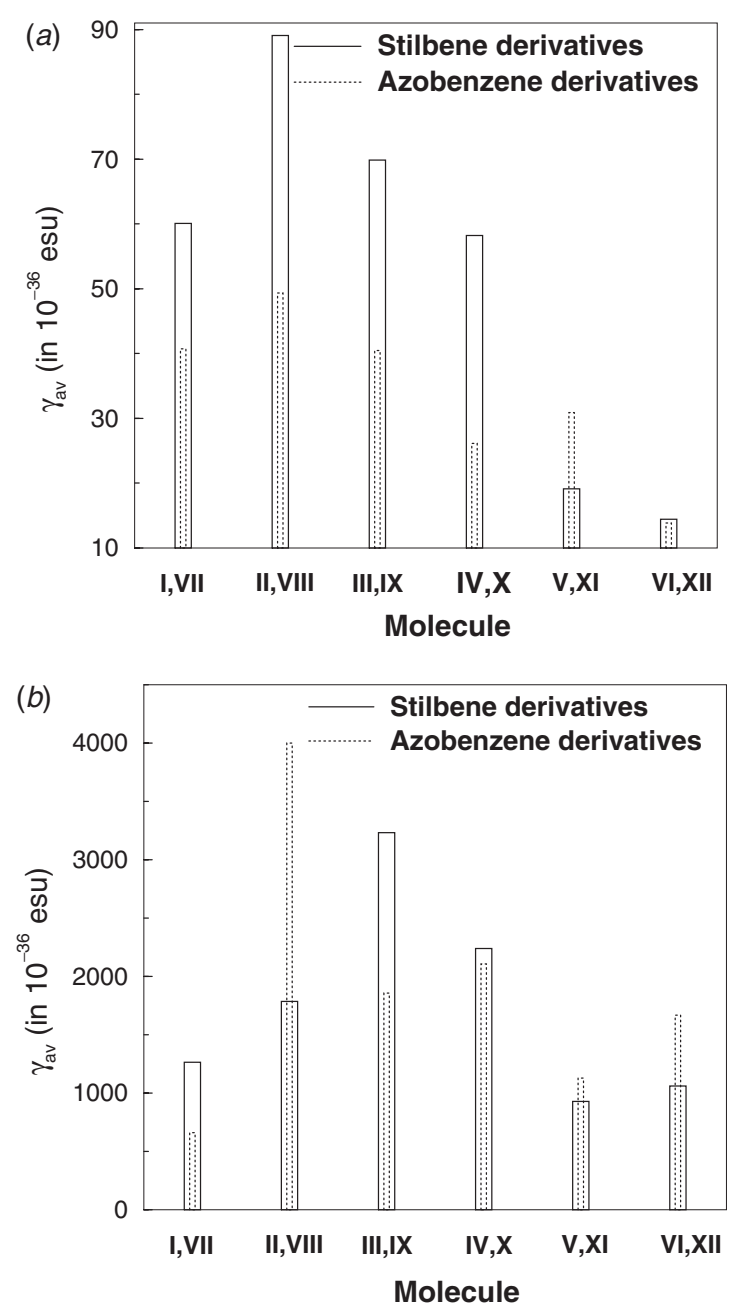

Figure 9. The orientationally averaged value of the THG coefficient $\left(\gamma_{\text {av }}\right)$ for stilbene and azobenzene derivatives at (a) $0.65 \mathrm{eV}$ and $(b) 1.17 \mathrm{eV}$.

In figure 9, we have presented the orientationally averaged third harmonic generation (THG) coefficient for our twelve molecules. We note that the THG coefficients are ten to fifty times larger at $1.17 \mathrm{eV}$ compared to those at $0.65 \mathrm{eV}$ excitation frequencies. The excitation energy of $1.17 \mathrm{eV}$ is very close to three-photon resonance, particularly in the trimethyl derivatives of azobenzene and this is reflected in the very large THG response of the molecules. However, the non-resonant THG response is very high in stilbene derivatives, particularly in the methyl susbtituted compounds. Quite unlike with the SHG coefficients, the di- and tri-phenyl compounds have a much smaller THG coefficient than the other derivatives. This is so in spite of the large conjugation lengths in the phenyl substituted compounds. This result shows that for the non-resonant THG coefficients, the electronic donoracceptor properties are more important than the size of the $\pi$-conjugation. All our molecules have non-zero dipole moment in the ground state. Therefore, an application of a static electric field will orient the molecules (partially) at room temperature, leading to breaking of the overall inversion symmetry of the randomly oriented systems. This will result in a nonzero second harmonic generation, which is different from the contribution due to the third-order nonlinear polarizability $\gamma_{\mathrm{e}}(-2 \omega ; \omega, \omega, 0)$. The total $\gamma_{\mathrm{t}}(-2 \omega ; \omega, \omega, 0)$ is given by

$$
\gamma_{\mathrm{t}}(-2 \omega ; \omega, \omega, 0)=\gamma_{\mathrm{e}}(-2 \omega ; \omega, \omega, 0)+\frac{\beta(-2 \omega ; \omega, \omega) \cdot \mu_{\mathrm{g}}}{5 k T},
$$

within a two-state model for the electric field induced second harmonic (EFISH) generation. From the computed $\beta_{\mathrm{av}}(2 \omega ; \omega, \omega)$ we have that it is possible to estimate the contribution to EFISH generation due to reorientation of the molecules in an electric field, since we know the ground state dipole moment. In table $7, \mu_{\mathrm{g}}$ and $\beta_{\mathrm{av}} \cdot \mu_{\mathrm{g}}$ for all the molecules we have studied are given.

We note that for corresponding substitutions, EFISH of stilbenes are higher than those for azobenzenes at $0.65 \mathrm{eV}$. It is important to mention that, although the EFISH value calculated here is modest as compared to recently reported thiophene based $\pi$-conjugated chromophores [42], they are among the highest for the cyclic and polymeric NLO active phosphazene compounds. At this point we stress that the most critical drawbacks in the implementation of a polymer-based electro-optic modulator for different applications has been the dearth of thermally stable nonlinear optical polymers. On the one hand, the thiophene based $\pi$-conjugated chromophores have the highest NLO coefficients among the cyclic and polymeric NLO active compounds, on the other hand they suffer from solubility problems. In this context, phenyl substituted azo-derivatized molecules can be a better substitute for the implementation of polymer-based electro-optic modulators as phosphorusnitrogen compounds have inherent thermal stability.

\subsection{Two-photon absorption cross-section}

Two-photon absorption cross-sections for the molecules in this study are given in table 8 for the three lowest excited states. Looking closely at table 8 one finds that the most dominating TPA state is not necessarily the first excited state. For all the molecules studied in this work, the most dominating TPA state is also the most dominating OPA state. These results are in conformity with the results of Agren and co-workers, i.e. for asymmetric molecules both the dominant OPA and the dominant TPA state are the same [29]. 
Table 8. Optical gap (in eV) and two-photon absorption cross-sections $(\mathrm{au})$ for the lowest three excited states for stilbene and azobenzen derivatives.

\begin{tabular}{|c|c|c|}
\hline Molecule & Optical gap & TPA \\
\hline I & $\begin{array}{l}3.64 \\
4.38 \\
4.59\end{array}$ & $\begin{array}{r}957.62 \\
9.15 \\
28.03\end{array}$ \\
\hline II & $\begin{array}{l}3.34 \\
4.12 \\
4.46\end{array}$ & $\begin{array}{r}1409.30 \\
0.09 \\
406.56\end{array}$ \\
\hline III & $\begin{array}{l}3.44 \\
4.12 \\
4.52\end{array}$ & $\begin{array}{r}1157.88 \\
0.14 \\
211.11\end{array}$ \\
\hline IV & $\begin{array}{l}3.40 \\
4.04 \\
4.45\end{array}$ & $\begin{array}{r}1362.16 \\
0.51 \\
11.77\end{array}$ \\
\hline V & $\begin{array}{l}3.32 \\
3.79 \\
4.30\end{array}$ & $\begin{array}{r}1006.77 \\
0.68 \\
33.09\end{array}$ \\
\hline VI & $\begin{array}{l}3.30 \\
4.32 \\
4.35\end{array}$ & $\begin{array}{r}1269.69 \\
10.45 \\
23.25\end{array}$ \\
\hline VII & $\begin{array}{l}3.28 \\
3.66 \\
4.42\end{array}$ & $\begin{array}{r}2.36 \\
637.60 \\
39.02\end{array}$ \\
\hline VIII & $\begin{array}{l}3.06 \\
3.47 \\
4.19\end{array}$ & $\begin{array}{r}14.62 \\
883.43 \\
0.11\end{array}$ \\
\hline IX & $\begin{array}{l}3.07 \\
3.58 \\
4.20\end{array}$ & $\begin{array}{r}8.77 \\
764.72 \\
0.02\end{array}$ \\
\hline $\mathrm{X}$ & $\begin{array}{l}3.00 \\
3.43 \\
4.04\end{array}$ & $\begin{array}{r}18.14 \\
1036.94 \\
3.51\end{array}$ \\
\hline XI & $\begin{array}{l}3.16 \\
3.42 \\
4.38\end{array}$ & $\begin{array}{r}43.77 \\
840.20 \\
21.71\end{array}$ \\
\hline XII & $\begin{array}{l}3.21 \\
3.42 \\
4.44\end{array}$ & $\begin{array}{r}53.03 \\
1163.44 \\
14.07\end{array}$ \\
\hline
\end{tabular}

In symmetric molecules, the dominant TPA state has the same symmetry as the ground state while the dominant OPA state has the opposite symmetry. Weak symmetry breaking removes the symmetry label of the state but retains largely the character of the OPA and the TPA states. Since in our case both the TPA and the OPA intensities are the largest for the same state, it implies that the derivatization has resulted in strong symmetry breaking of the states of parent backbone molecule. For the stilbene backbone, it is the first excited state, $S_{1}$, that is TPA allowed and for the azobenzene

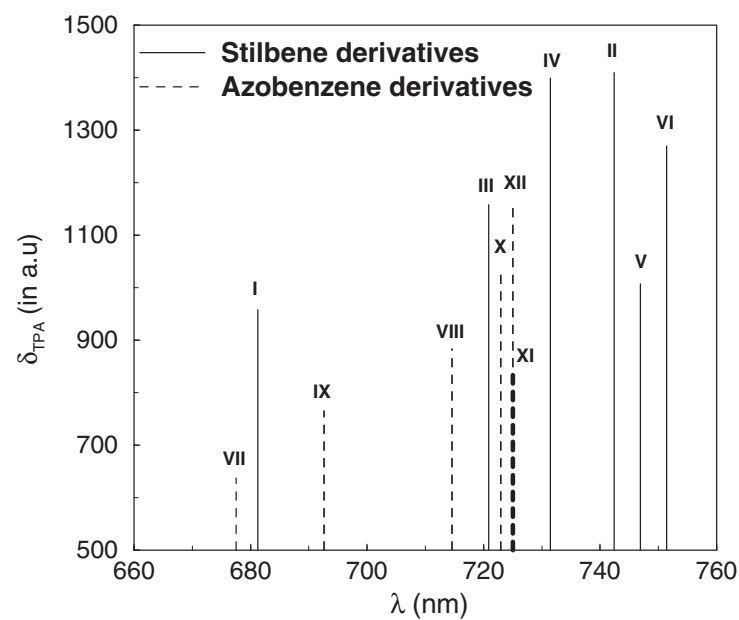

Figure 10. Two-photon absorption cross-section of the dominant TPA state for stilbene and azobenzene derivatives.

backbone molecules, it is the second excited state, $S_{2}$. For azobenzene derivatives, the TPA as well as OPA cross-section for the $S_{1}$ state is negligible. Here again we find that the TPA of stilbene derivatives is larger than those of azobenzene derivatives. In the stilbene derivatives, methyl substitution as well as single phenyl substitution at the phosphorus site leads to the largest TPA, while triphenyl substitution leads to largest $\delta_{\text {tpa }}$ in azobenzene derivatives (see figure 10). Thus, attaching a strong donor group to the phosphorus site seems to enhance $\delta_{\text {tpa }}$ in both azobenzene and stilbene derivatives.

\section{Conclusion}

In this study we have demonstrated that the $\mathrm{R}_{3}-\mathrm{P}=\mathrm{N}$ group can be used as an efficient donor nonlinear chromophore with stilbene and azobenzene $\pi$-backbones. We find that both systems have similar optical gaps, if we focus on the strongly allowed excitation which for stilbenes is the first excited singlet state and for azobenzenes the second excited singlet state. The linear polarizability and the SHG coefficient are large for the triphenyl derivatives and least for the triamino derivatives. These are not strongly frequency dependent up to $1.17 \mathrm{eV}$ excitation frequency. The monophenyl derivative has both $\alpha_{\mathrm{av}}$ and $\left|\beta_{\mathrm{av}}\right|$ values which are comparable to the diphenyl and triphenyl derivatives. The THG coefficient is closer to resonance at $1.17 \mathrm{eV}$. The low frequency THG coefficient is largest for the trimethyl derivative both for stilbenes and azobenzenes, although, the former have larger values for the same derivatization. At $1.17 \mathrm{eV}$ all the THG coefficients are ten to fifty times larger than those at $0.65 \mathrm{eV}$. The trimethyl 
azobenzene derivative shows the largest increase as it is closest to three-photon resonance. The EFISH coefficients arising due to alignment of the dipole is also large but not as large as that of thiophenes. The TPA in all these systems is strongest to the state to which OPA is also strong. This suggests strong asymmetry of the system. The TPA cross-section is larger for stilbene derivatives than for the azobenzene derivatives and trimethyl derivatized stilbene has the largest TPA crosssection among the systems we have studied. The high thermal stability and the ease of chemical manipulation together with large NLO responses make the azaphosphane derivatives linked to the stilbene backbone promising candidates for organic optical devices.

\section{Acknowledement}

This work is partly supported by the Board for Research in Nuclear Science through its grant no. 99/ 3/37/BRNS/735.

\section{References}

[1] T.J. Skotheim. Handbook of Conducting Polymers, Dekker, New York (1986) (see also references therein).

[2] D.S. Chemla, J. Zyss. Nonlinear Optical Properties of Organic Molecules and Crystals, Academic Press, New York (1987).

[3] P.N. Prasad, D.J. Williams. Introduction to Nonlinear Optical Effects in Molecules and Polymers, John Wiley, New York (1991).

[4] H.S. Nalwa, M. Siezo. Nonlinear Optics of Organic Molecules and Polymers, CRC Press, Boco Raton, FL (1994).

[5] J. Zyss. Molecular Nonlinear Optics: Materials, Physics and Devices, Academic Press, New York (1994).

[6] L.R. Dalton. Nonlinear Optical Polymeric Materials: From Chromophore Design to Commercial Applications, in the series Advances in Polymer Science, Vol. 158, p. 1, Springer-Verlag, Heidelberg (2001).

[7] L.R. Dalton. The Role of Nonlinear Optical Devices in the Optical Communications Age, Kluwer Academic Publishers, Dordrecht (2001).

[8] Y.V. Pereverev, O.V. Prezhdo, L.R. Dalton. Chem. Phys. Lett., 340, 328 (2001).

[9] S.P. Karna, Y. Zhang, M. Samoc, P.N. Prasad, B.A. Reinhardt, A.G. Dillard. J. chem. Phys., 90, 9984 (1993).

[10] H. Ma, B. Chen, T. Sassa, L.R. Dalton, A.K. Yen. J. Am. chem. Soc., 123, 986 (2001).

[11] H. Ma, J. Wu, P. Herguth, B. Chen., A.K. Jen. Chem. Mater., 12, 1187 (2000).

[12] C.R. Moylan, S. Ermer, S.M. Lovejoy, I.H. McComb, D.S. Leung, R. Wortmann, P. Krdmer, R.J. Tweig. J. Am. chem. Soc., 118, 12950 (1996).
[13] B.R. Cho, S.J. Lee, S.H. Lee, K.H. Son, Y.H. Kim, J.Y. Doo, G.J. Lee, T.I. Kang, Y.K. Lee, M. Cho, S.J. Jeon. Chem. Mater., 13, 1438 (2001).

[14] J.M. Fox, T.J. Katz, S.V. Elshocht, T. Verbiest, M. Kauranen, A. Persoons, T. Thongpanchang, T. Kraus, L. Brus. J. Am. chem. Soc., 121, 3453 (2001).

[15] T. Verbiest, S.V. Elshocht, M. Kauranen, L. Hellamans, J. Snauwaert, C. Nuckolls, T.J. Katz, A. Persoons. Science, 282, 913 (1998).

[16] N.J. Long. Angew. Chem. Int. Ed. Engl., 34, 21 (1995).

[17] S.R. Marder, D.N. Beratan, L. Cheng. Science, 252, 103 (1991); M. Stahelin, D.M. Burland, J.E. Rice. Chem. Phys. Lett., 191, 245 (1992).

[18] S. Ramasesha, P.K. Das. Chem. Phys., 145, 343 (1990).

[19] B.L. Davydov, L.D. Derkacheva, V.V. Dunina, M.E. Zhabotinskii, V.F. Zolin, L.G. Koreneva, M.A. Samokhina. Opt. Spectrosc. Engl. Trans., 30, 503 (1971); B.L. Davydov, V.V. Dunina, V.F. Zolin, L.G. Koreneva. ibid., 34, 267 (1973).

[20] J.L. Oudar, D.S. Chemla. J. Chem. Phys., 66, 2664 (1977).

[21] D.R. Kanis, M.A. Ratner, T.J. Marks. Chem. Rev., 94, 195 (1994); S.R. Marder, J.W. Perry. Adv. Mater., 5, 804 (1993); T.J. Marks, M.A. Ratner. Angew. Chem. Int. Ed. Engl., 34, 155 (1995); Y. Shi, C. Zhang, J.H. Bechtel, L.R. Dalton, B.H. Robinson, W.H. Steier. Science, 288, 119 (2000).

[22] K.V. Katti, K. Raghuraman, N. Pillarsetty, S.R. Kara, R.J. Gaulotty, M.A. Chartier, C.A. Langhoff. Chem. Mater., 14, 2436 (2002).

[23] H.R. Allcock, A.A. Dembeck, C. Kim, R.L.S. Devine, Y. Shi, W.H. Steier, C.W. Spangler. Macromolecules, 24, 1000 (1991); Chem. Mater., 2, 97 (1990).

[24] K.V. Katti, R.G. Cavell. Comments Inorg. Chem., 10, 53 (1990); E.W. Abel, S.A. Mucklejohn. Phosphorus Sulfur, 9, 235 (1981); K.V. Katti, P.R. Singh, K.K. Katti, C.L. Barnes, K. Kopica, A.R. Ketring, W.A. Volkert. Phosphorus Sulfur Relat. Elem., 75, 55 (1993).

[25] J. Ridley, M.C. Zerner. Theor. Chim. Acta, 32, 111 (1973); A.D. Bacon, M.C. Zerner. Theor. Chim. Acta, 53, 21 (1979).

[26] S. Ramasesha, Z. Shuai, J.L. Bredas. Chem. Phys. Lett., 245, 224 (1995).

[27] Z.G. Soos, S. Ramasesha. J. chem. Phys., 90, 1067 (1989).

[28] S. Ramasesha. J. comput. Chem., 11, 545 (1990).

[29] C.K. Wang, P. Macak, Y. Luo, H. Agren. J. Chem. Phys., 114, 9813 (2001).

[30] M.J.S. Dewar, E.G. Zoebisch, E.F. Healy, J.J.P. Stewart. J. Am. chem. Soc., 107, 3902 (1985).

[31] M.J. Frisch, G.W. Trucks, H.B. Schlegel, G.E. Scuseria, M.A. Robb, J.R. Cheeseman, V.G. Zakrzewski, J.A. Montgomery Jr, R.E. Stratmann, J.C. Burant, S. Dapprich, J.M. Millam, A.D. Daniels, K.N. Kudin, M.C. Strain, O. Farkas, J. Tomasi, V. Barone, M. Cossi, R. Cammi, B. Mennucci, C. Pomelli, C. Adamo, S. Clifford, J. Ochterski, G.A. Petersson, P.Y. Ayala, Q. Qui, K. Morokuma, P. Salvador, J.J. Dannenberg, D.K. Malik, A.D. Rabuck, K. Raghavachari, J.B. Foresman, J. Cioslowski, J.V. Ortiz, A.G. Baboul, B.B. Stefanov, G. Liu, A. Liashenko, P.Piskorz, I. Komaromi, R. Gomperts, R.L. Martin, D.J. Fox, T. Keith, M.A. Al-Laham, C.Y. Peng, A. Nanayakkara, M. Challakombe, P.M.W. Gill, B. Johnson, W. Chen, M.W. Wong, J.L. Andres, C. Gonzalez, M. Head-Gordon, E.S. Replogle, J.A. Pople. Gaussian 98, revision A.10, Gaussian, Inc., Pittsburgh, PA (2001). 
[32] C.J. Brown. Acta Crystallogr., 21, 146 (1966); J.A. Bouwstra, A. Scuouten, J. Kroon. Acta Crystallogr. C, 39, 1121 (1983).

[33] P.C. Chen, Y.C. Chieh. J. molec. Struct. (Theochem), 624, 191 (2003); V. Stepanic, G. Baranovic, V. Smrecki. J. molec. Struct., 569, 89 (2001).

[34] J.O. Morley, P. Paldives, D. Pugh. J. chem. Phys., 99, 5197 (1993); J.O. Morley. J. molec. Struct. (Theochem), 362, 235 (1996).

[35] J.O. Morley. J. molec. Struct. (Theochem), 340, 45 (1995).

[36] R. Keat. Organophosphorus Chem. (Roy. Soc. Chem. Lond.), 6, 182 (1974); ibid., 7, 188 (1977); ibid., 8, 204 (1978); ibid., 9, 210 (1979); ibid., 10, 232 (1980); ibid., 11, 219 (1980); J.C. Clardy, R.L. Kolpa, J.G. Verkade. Phosphorus, 4, 133 (1974).
[37] L.-T. Cheng, W. Tam, S.R. Marder, A.E. Stiegman, G. Rikken, C.W. Spangler. J. phys. Chem., 95, 10643 (1991).

[38] C.L. Liu, Z.M. Su, J.K. Feng, A.M. Ren, C.C. Sun, Z.Z. Zha, Q. Wang. J. molec. Struct. (Theochem), 531, 169 (2000).

[39] F. Meyers, S.R. Marder, B.M. Pierce, J.L. Bredas. J. Am. Chem. Soc., 116, 10703 (1994).

[40] P. Romaniello, F. Lelj. J. fluor. Chem., 125, 145 (2004).

[41] A. Ulman, C.S. Willand, W. Kohler, D.R. Robello, D.J. Williams, L. Handley. J. Am. chem. Soc., 112, 7083 (1990).

[42] J. Raimundo, P. Blanchard, N. Gallego-Planas, N. Mercier, I. Ledoux-Rak, R. Hierle, J. Roncali. J. org. Chem., 67, 205 (2002). 